\title{
A aliança entre Tecnologias do passado e Tecnologias Digitais da Informação e Comunicação via Investigação Científica
}

\author{
Gisele Pereira Oliveira1 ${ }^{1}$ \\ Universidade Estadual do Ceará (UECE), Centro de Educação, Programa de Pós-graduação em \\ Educação, Fortaleza, Ceará, Brasil
}

Ana Carolina Costa Pereira ${ }^{2}$ (D)

Universidade Estadual do Ceará (UECE), Programa de Pós-graduação em Educação, Departamento de Educação e Matemática, Fortaleza, Ceará, Brasil

\begin{abstract}
Resumo
O século XXI tem sido reconhecido pelo uso frequente de Tecnologias Digitais da Informação e Comunicação (TDIC), pela divulgação de informação e comunicação em rápida velocidade, cenário que nos inquieta a perceber que os recursos tecnológicos já estavam presentes em séculos passados, com funcionalidades, interfaces, contextos históricos e necessidades distintas em cada período. Diante dessa compreensão, desenvolvemos esse estudo com o objetivo geral de compreender a aliança entre as tecnologias do passado e as tecnologias digitais via investigação científica como meio favorável ao ensino e aprendizagem da Matemática. Buscando-se alcançar tal objetivo, o percurso metodológico desta foi pautado conforme a Engenharia Didática de segunda geração, organizada em quatro fases: as análises preliminares, concepção e análise a priori das situações didáticas, experimentação e análise a posteriori validação. Resultou-se mediante ao uso da plataforma Storyboard That, a produção de uma história em quadrinhos, que tratou introdutoriamente sobre fatos presentes na história da Matemática, fazendo a utilização do instrumento náutico/matemático, balhestilha, de Manoel de Figueiredo, de 1603, contido no documento histórico, Chronographia Reportório dos Tempos[...], em que através dos quadrinhos estabeleceu a aliança entre as tecnologias do passado e as digitais, obtendo um recurso atrativo e lúdico, que pode ser relevante ao ensino de saberes matemáticos. Dessa maneira, concluímos que a articulação entre tecnologias de períodos distintos no ensino de Matemática é possível, mas mediante a realização de um tratamento didático, que destaque a intencionalidade da ação, assim como a construção de um plano de ação que contenha uma metodologia norteadora e recursos educacionais digitais.
\end{abstract}

Submetido em: 07/01/2021

Aceito em: 03/07/2021

Publicado em: 20/07/2021

\footnotetext{
${ }^{1}$ Doutoranda em Educação Matemática pela Universidade Estadual do Ceará (UECE). Professora de Matemática da educação básica na Secretaria de Educação do Estado do Ceará (SEDUC). Endereço para correspondência: E-mail: gisele.oliveira@aluno.uece.br.

2 Doutora em Educação Matemática pela Universidade Federal do Rio Grande do Norte (UFRN). Professora da licenciatura em Matemática e da Pós-graduação em Educação pela Universidade Estadual do Ceará; Professora do Programa de Pós-graduação em Ensino de Ciências e Matemática do Instituto Federal de Educação, Ciência e Tecnologia do Ceará (IFCE). Endereço para correspondência: E-mail: carolina.pereira@uece.br.
} 
eISSN: $2526-9062$

Palavras-chave: Tecnologias do Passado; Tecnologias Digitais da Informação e Comunicação; Investigação Científica.

\title{
The alliance between Technologies of the past and Digital Technologies of Information and Communication via Scientific Research
}

\begin{abstract}
The 21st century has been recognized for the frequent use of Digital Technologies of Information and Communication (TDIC), for the dissemination of information and communication at fast speed, a scenario that makes us uneasy to realize that technological resources were already present in past centuries, with functionalities, interfaces, historical contexts and distinct needs in each period. Given this understanding, we developed this study with the general objective of understanding the alliance between past technologies and digital technologies via scientific investigation as a favorable means for teaching and learning Mathematics. Seeking to achieve this objective, the methodological path of this was based on second-generation Didactic Engineering, organized into four phases: preliminary analysis, conception and a priori analysis of didactic situations, experimentation and analysis a posteriori validation. It resulted through the use of the Storyboard That platform, the production of a comic book, which dealt introductory with facts present in the history of Mathematics, making use of the nautical/mathematical instrument, balhestilha, by Manoel de Figueiredo, from 1603, contained in the historical document, Chronographia Reportório dos Tempos[...], in which he established an alliance between past and digital technologies through comic strips, obtaining an attractive and playful resource, which can be relevant to the teaching of mathematical knowledge. Thus, we conclude that the articulation between technologies from different periods in the teaching of Mathematics is possible, but through the realization of a didactic treatment, which highlights the intention of the action, as well as the construction of an action plan that contains a guiding methodology and digital educational resources.
\end{abstract}

Keywords: Technologies of the Past; Digital Information and Communication Technologies; Scientific investigation.

\section{La alianza entre las tecnologías del pasado y las tecnologías digitales de la información y la comunicación a través de la investigación científica}

\begin{abstract}
Resumen
El siglo XXI ha sido reconocido por el uso frecuente de las Tecnologías Digitales de Información y Comunicación (TDIC), para la difusión de información y comunicación a gran velocidad, escenario que nos incomoda darnos cuenta de que los recursos tecnológicos ya estaban presentes en siglos pasados, con funcionalidades, interfaces, contextos históricos y necesidades distintas en cada período. Dado este entendimiento, desarrollamos este estudio con el objetivo general de comprender la alianza entre tecnologías pasadas y tecnologías digitales a través de la investigación científica como un medio favorable para la enseñanza y el aprendizaje de las Matemáticas. Buscando alcanzar este objetivo, la trayectoria metodológica de esta se basó en la Ingeniería Didáctica de segunda generación, organizada
\end{abstract}


en cuatro fases: análisis preliminar, concepción y análisis a priori de situaciones didácticas, experimentación y análisis a posteriori validación. El resultado fue la utilización de la plataforma Storyboard That, la producción de un cómic, que abordó de manera introductoria los hechos presentes en la historia de las Matemáticas, haciendo uso del instrumento náutico / matemático, balhestilha, de Manoel de Figueiredo, de 1603, contenido en el documento histórico Chronographia Reportório dos Tempos, [...] en el que establece una alianza entre el pasado y las tecnologías digitales a través de las historietas, obteniendo un recurso atractivo y lúdico, que puede ser relevante para la enseñanza del conocimiento matemático. Así, concluimos que la articulación entre tecnologías de diferentes épocas en la enseñanza de las Matemáticas es posible, pero a través de la realización de un tratamiento didáctico, que resalte la intención de la acción, así como la construcción de un plan de acción que contenga una guía. metodología y recursos educativos digitales.

Palabras clave: Tecnologías del pasado; Tecnologías de la información y las comunicaciones digitales; Investigación científica.

\section{Introdução}

Estamos imersos em um momento que as informações e comunicações estão disponíveis e divulgadas numa rápida velocidade, período esse reconhecido como a era tecnológica, em que mediante ao uso de Tecnologias da Informação e Comunicação (TIC) e as Tecnologias Digitais da Informação e Comunicação (TDIC), vários ambientes, instituições promotoras de educação e práticas sociais, acadêmicas e outras, vêm sendo facilitadas e expandidas.

E, diante deste contexto, debruçamo-nos a refletir sobre distintas situações, que requerem uma formação inicial e/ou continuada de professores sobre estes recursos, podendo perceber, conforme Pinheiro, Pedrosa e Mendonça (2016), a importância de refletir sobre como e quando inserir esses em circunstâncias promotoras de ensino e aprendizagem, reconhecendo e utilizando os potenciais fornecidos através do uso destes.

Nessa compreensão, ademais, em especial no cenário educacional, um contraponto a essa realidade, que ainda que estejamos participando e vivenciando rotinas com o uso de TIC e TDIC, nos inquieta observar que ainda existe grande fragilidade na formação inicial e continuada de professores, nos variados níveis de ensino.

Com esse intuito, também, saberes do conhecimento poderiam ser alcançados a partir de um uso didático destes, expondo-se à atender a necessidade de várias disciplinas, como por exemplo, a Matemática, que é apontada por muitos como "difícil”, mas que na maioria das vezes, através da utilização pedagógica e metodológica, ofertam possivelmente a desmitificação de tais percepções. 
eISSN: $2526-9062$

No mais, tem-se visto, a partir da observação do cenário da educação brasileira, a ausência de assertividade quanto ao tratamento didático atribuído à tais ferramentas, desperdiçando assim, possibilidades de nessa trajetória direcionada, vislumbrar a execução de significativas práxis docentes, que poderiam se concretizar, contribuindo com a aprendizagem de alunos, caso fossem realizadas iniciativas, rumo a formação destes professores, em relação ao planejamento de ações com a inserção de tais recursos e a constituição de perspectivas de usabilidade das TDIC no ensino de Matemática.

Em continuidade à discussão desse estudo, tem-se documentos oficiais reconhecidos em todo o território nacional brasileiro, como a Base Nacional Comum Curricular (BNCC), os Parâmetros Curriculares Nacionais (PCNs), as Diretrizes Curriculares Nacionais (DCNs) e outros, que sugerem, orientam e solicitam o uso de tecnologias no ensino das variadas ciências.

Diante disso, visualizou-se na BNCC, consoante Brasil (2018), os desígnios para educação, da integralização das TDIC na Linguagens e suas tecnologias, Ciências Humanas e suas tecnologias, Ciências da Natureza e suas tecnologias e Matemática e suas tecnologias, como áreas amparadas pelo uso de tecnologias no contexto educacional e na emersão dos saberes do conhecimento.

Desse modo, na Matemática e suas tecnologias, conforme Brasil (2018), verificou-se a possibilidade potente de uso da história da Matemática no processo para "aprender a aprender" saberes, que não tratam do cotidiano do século XXI, mas que podem ser submetidos a uma transposição didática, mediante, à um planejamento eficaz, resultante assim, numa aliança entre recursos presentes na história da Matemática e nas TDIC, via investigação científica.

Esses recursos, podem-se conhecer em séculos passados, como instrumentos, ferramentas e utensílios capazes de modificar o ambiente/meio, assim como o próprio indivíduo em suas tarefas cotidianas, em suas respectivas épocas, favorecendo e otimizando os ofícios desenvolvidos por estes.

Para Veraszto et al. (2009), no passado, já existiam tecnologias, pois referiam-se aquilo que poderia facilitar e transformar a vida do homem, sendo por exemplo, considerados tecnologias no século XVI, os instrumentos matemáticos, que segundo Saito (2015) são ferramentas concebidas para medir, o que Aristóteles já denominava por quantidade, sendo usados para muitas finalidades e 
eISSN: $2526-9062$

ofícios, como navegação, pintura, escultura, agrimensura, arquitetura, astronomia e outras atividades desenvolvidas pelo praticantes das matemáticas ${ }^{3}$.

E, assim, neste estudo, enxergamos a possibilidade de através de um tratamento didático, estabelecer uma aliança entre as tecnologias do passado, os instrumentos, e, as TDIC no ensino de Matemática, promovida via investigação científica de materiais, plataformas, recursos presentes no meio acadêmico.

Para facilitar a trajetória nesse contexto, estabelecemos como norteadora das ações, a pergunta diretriz, "Como as tecnologias do passado e as Tecnologias Digitais da Informação e Comunicação via investigação científica podem colaborar com o ensino de Matemática?”.

E, dessa maneira, buscando levantar respostas para essa questão de pesquisa, estabeleceu-se o objetivo geral de compreender a aliança entre as tecnologias do passado e as tecnologias digitais via investigação científica como meio favorável ao ensino e aprendizagem de Matemática.

\section{A aliança entre tecnologia do passado e Tecnologias Digitais da Informação e Comunicação}

$\mathrm{Na}$ busca por promover uma aliança entre as tecnologias do passado, os instrumentos matemáticos e as TDIC, inquietamo-nos a compreender, os cenários particulares de tais contextos e mediante a investigação científica de evidências, buscou-se amparar e sustentar essa relação significativa de recursos, para oferecer o desenvolvimento de competências e habilidades educacionais

E, assim, de acordo com Rodrigues (2001) compreendemos a natureza da etimologia da palavra tecnologia e o contexto que essa representa, pois provém da junção do termo tecno, do grego techné, que significa saber fazer e logia, do grego que corresponde a razão. Com isso, tecnologia compreende-se por ser a razão do saber fazer. E, segundo Veraszto et al. (2009):

A tecnologia exige um profundo conhecimento do porquê e do como seus objetivos são alcançados, se constituindo em um conjunto de atividades humanas associadas a um sistema de símbolos, instrumentos e máquinas, e assim, visa a construção de obras e a fabricação de produtos, segundo teorias, métodos e processos da ciência moderna.

\footnotetext{
${ }^{3}$ Para Saito (2018) os Praticantes das Matemáticas eram arquitetos, pintores, escultores, navegadores, agrimensores e outros que fabricavam instrumentos e escreviam tratados sobre seus ofícios, buscando transmitir seus conhecimentos a novas gerações, valorizando sua arte e se autopromovendo.
} 
eISSN: $2526-9062$

Em que, nesta compreensão, percebe-se que a tecnologia, seja do passado, como os instrumentos matemáticos, ou às TDIC, neste século, se assemelham em algumas características no sentido de serem usadas nos contextos e períodos distintos para favorecer a vida humana, com suas interfaces de representação, simbologia, instrumentalização, fabricação, métodos e processos favoráveis ao ensino de Matemática.

E, nessa direção, Veraszto et al. (2009) reflete que existe muitas correspondências nas definições de ciência e tecnologia, em que, respectivamente, a primeira entende-se por reflexão, descrição e explicação de fenômenos naturais através de problemas e outras circunstâncias; enquanto a segunda, fabrica o artificial por meio da formulação e seleção de ideias entre outros elementos. Assim, visualizamos que as tecnologias possuem um viés mais voltado para o utilitário, o facilitador, promotor de transformação, favorável à aplicação.

Já as TICs, consoante a Fiorentini e Lorenzato (2007) são resultantes das tecnologias da informação, identificadas por informática, e as tecnologias da comunicação, aquelas ditas de cunho das telecomunicações e mídia eletrônica. E, também correspondentes e semelhantes, as TDIC, que conforme Souza (2020) são recursos digitais favoráveis ao uso no ensino em ambiente educacional digital.

Diante disso, por intermédio ao destaque das tecnologias, em períodos distintos, possuindo intenções correspondentes, destacamos que a aliança, vislumbrada neste estudo, constitui-se a partir da investigação científica de literatura nesta abordagem, recursos, metodologias e reflexões, que levanos à crer na possibilidade de articulação entre tecnologias do passado e TDIC.

Com isso, podem ser capazes de serem sustentadas pelo tripé construído neste percurso, isto é, planejamento, metodologias e recursos, levando continuamente em consideração que a articulação de materiais e afins de momentos distintos não é fácil, mas que é possível através de tratamento didático.

Nessa aliança o tratamento didático é parte fundante do processo de articulação entre tecnologias do passado e TDIC, podendo perceber, via investigação científica, que os instrumentos matemáticos, tecnologias do passado presentes na história da Matemática, são reconhecidos, à maioria destes, a partir de Saito e Pereira (2019), em documentos históricos de períodos passados, requerendo inicialmente, como momento desse tratamento, a familiarização do documento, o 
eISSN: $2526-9062$

reconhecido de objetivos, público destinado e outras informações que sejam relevantes ao manuseio e compreensão destes.

Em seguida, Saito e Pereira (2019) alegam que esse tratamento solicita estabelecer uma intencionalidade de aprendizagem, isto é, o que se pretende na utilização destes, mediante a observação e planejamento de ações, na perspectiva de emergir potencialidade didática nesse processo. E, de posse deste reconhecimento e intencionalidade, caminha-se para a confecção de um plano de ação.

E, portanto, nessa construção didática, pedagógica e metodológica, passa-se a vislumbrar um cenário de articulação, relação e aliança entre as tecnologias do passado e as TDIC, em que submetidas a uma transposição didática, podem ser relacionadas, como exemplo, em recursos como os Objetos de Aprendizagem (OA), que mediante Wiley (2000), diz respeito à recursos digitais, que podem ser reutilizados em variadas situações, amparando à aprendizagem e Castro Filho et al. (2008) reforça que são recursos educacionais digitais, disponíveis na internet, com pouca duração para a usabilidade em sala de aula.

Desse modo, a aliança é fortalecida via investigação científica, permitindo compreender e identificar o melhor percurso neste tratamento didático, capaz de estabelecer a relação, interação e interface entre tecnologias do passado, os instrumentos matemáticos, presentes na história da Matemática, conforme percebidos em Batista (2018) e Batista e Pereira (2020), com a balhestilha de Manoel de Figueiredo (1603), em Alves (2019), com o círculo de proporção de William Oughtred (1633), em Oliveira (2019) e Oliveira e Pereira (2020), com jacente no plano de Pedro Nunes (1566), em Martins (2019), com as barras de calcular de John Napier (1617), em Albuquerque (2019), com o traité de Gerbert (1843), Silva e Pereira (2020) e Pereira e Saito (2019) com o Báculo de Petrus Ramus, Santos, Oliveira e Pereira (2020) com a régua de cálculo, e as do século XXI, as TDIC, consoante ressaltados por Souza (2020).

\section{Percurso Metodológico}

Esse estudo foi organizado conforme a engenharia didática de segunda geração, se propondo, realizar as fases que essa orienta, isto é, análises preliminares, concepção e análise a priori das situações didáticas, experimentação e análise a posteriori validação da pesquisa. Em que, segundo Artigue (1989) apud Almouloud e Silva (2012, p.26): 
eISSN: $2526-9062$

[...] 1. Análises preliminares: considerações sobre o quadro teórico didático geral e os conhecimentos já adquiridos sobre o assunto em questão [...]. 2. Concepção e análise a priori das situações didáticas: o pesquisador, orientado pelas análises preliminares, delimita certo número de variáveis pertinentes ao sistema sobre os quais o ensino pode atuar chamadas de variáveis de comando (microdidáticas ou macrodidáticas). 3. Experimentação: consiste na aplicação da sequência didática [...]. 4. Análise a posteriori e validação: A análise a posteriori consiste em uma análise de um conjunto de dados colhidos ao longo da experimentação, como por exemplo, produção dos alunos, registros de observadores e registro em vídeo. Nessa análise, se faz necessário sua confrontação com a análise a priori para que seja feita a validação ou não das hipóteses formuladas na investigação.

E, diante desse contexto, na fase de Análise Preliminar, nos debruçamos a desenvolver essa pesquisa, conduzidos inicialmente a realizar um levantamento bibliográfico de estudos e ferramentas, que fornecessem um ambiente fértil para a aliança entre as tecnologias do passado e as TDIC. Essa fase de investigação científica realizou-se, com estudo de recursos identificados em plataformas nacionais e internacionais de uso e produção de OAs, como o Banco Internacional de Objetos Educacionais $(\mathrm{BIOE})^{4}$, Rede Interativa Virtual de Educação (RIVED) ${ }^{5}$, Portal do Professor ${ }^{6}$, Pixton ${ }^{7}$ e o Storyboard That ${ }^{8}$.

Em seguida, ainda na primeira fase, realizamos uma investigação na plataforma $J S T O R^{9}$ sobre instrumentos matemáticos, para coletar literaturas e evidências que nos amparassem. Em que, através da submissão na busca deste, da palavra-chave "Mathematical Instrument", foram identificados alguns materiais de suporte para serem usados em outras ocasiões, durante pesquisa doutoral do Programa de Pós-graduação em Educação (PPGE) da Universidade Estadual do Ceará (UECE).

Vale ressaltar, que para esse estudo, restringimo-nos selecionar apenas as imagens, identificadas no JSTOR, destacando-se três imagens relevantes, de um total de oito levantadas, que apresentaram em suas representações e interface a constituição de fundamentos sobre a importância das tecnologias do passado, os instrumentos matemáticos, em seus períodos.

Em seguimento, na Concepção e Análise a priori das situações didáticas, planejamos as ações à serem desenvolvidas neste, estabelecendo às variáveis norteadoras e de investigação, como a

\footnotetext{
${ }^{4}$ BIOE: < http://objetoseducacionais.mec.gov.br/\#/inicio $>$.

${ }^{5}$ RIVED: $\langle$ http://www.dmm.im.ufrj.br/projeto/rived/index.html $>$.

${ }^{6}$ Portal do Professor: < http://portaldoprofessor.mec.gov.br/index.html $>$.

${ }^{7}$ PIXTON: < https://www.pixton.com/br/>.

${ }^{8}$ Storyboard That: < https://www.storyboardthat.com/ $>$.

${ }^{9}$ JSTOR: < https://www.jstor.org/>.
} 
eISSN: $2526-9062$

pergunta diretriz, objetivo geral, ferramentas selecionadas para experimentação e visualização da aliança entre tecnologias do passado e TDIC.

Em continuidade, na fase de Experimentação, selecionamos o aplicativo Storyboard That, identificado na primeira fase no levantamento nas plataformas e selecionado na fase seguinte para uso nesta, tendo a produção de um Storyboard ${ }^{10}$ pelas autoras desse estudo, denotado por história em quadrinhos Navemática (ver figura 01), conforme orientado na engenharia didática de segunda geração, que aponta a necessidade da produção de um OA para uso na formação de professores ou para o ensino regular, em que neste estudo articulou uma tecnologia do passado com as TDIC.

Neste momento, selecionou-se a balhestilha, contida no documento histórico Chronograhia Reportório dos Tempos [...] de Manoel de Figueiredo, de 1603, que é um instrumento náutico e matemático, identificado na dissertação de mestrado de Batista (2018), mediante aos achados preliminares conforme Engenharia Didática.

Essa aliança pretendida foi estabelecida na transposição didática do instrumento balhestilha para o ambiente digital, desmitificando sua apresentação tradicional em livros e produções acadêmicas, para o cenário do Storyboard, isto é, a breve História em quadrinhos, que oportuniza o lúdico, criativo e inovador em suas formas, cores e conexões, e, a aliança entre a tecnologia do século XVII, o instrumento balhestilha e as TDIC, no ambiente da História em quadrinhos, fornecidos pelo Storyboard That (ver Figura 01).

\footnotetext{
${ }^{10}$ Storyboard é um roteiro de produção de cenas para OA, filmes, livros e outros meios de informação e comunicação, em que através de uma sequência cronológica de desenhos, como em história em quadrinhos, apresentam-se a composição contínua de cenas, que ao final compõem um produto.
} 
eISSN: $2526-9062$

Figura 01- História em Quadrinhos Navemática
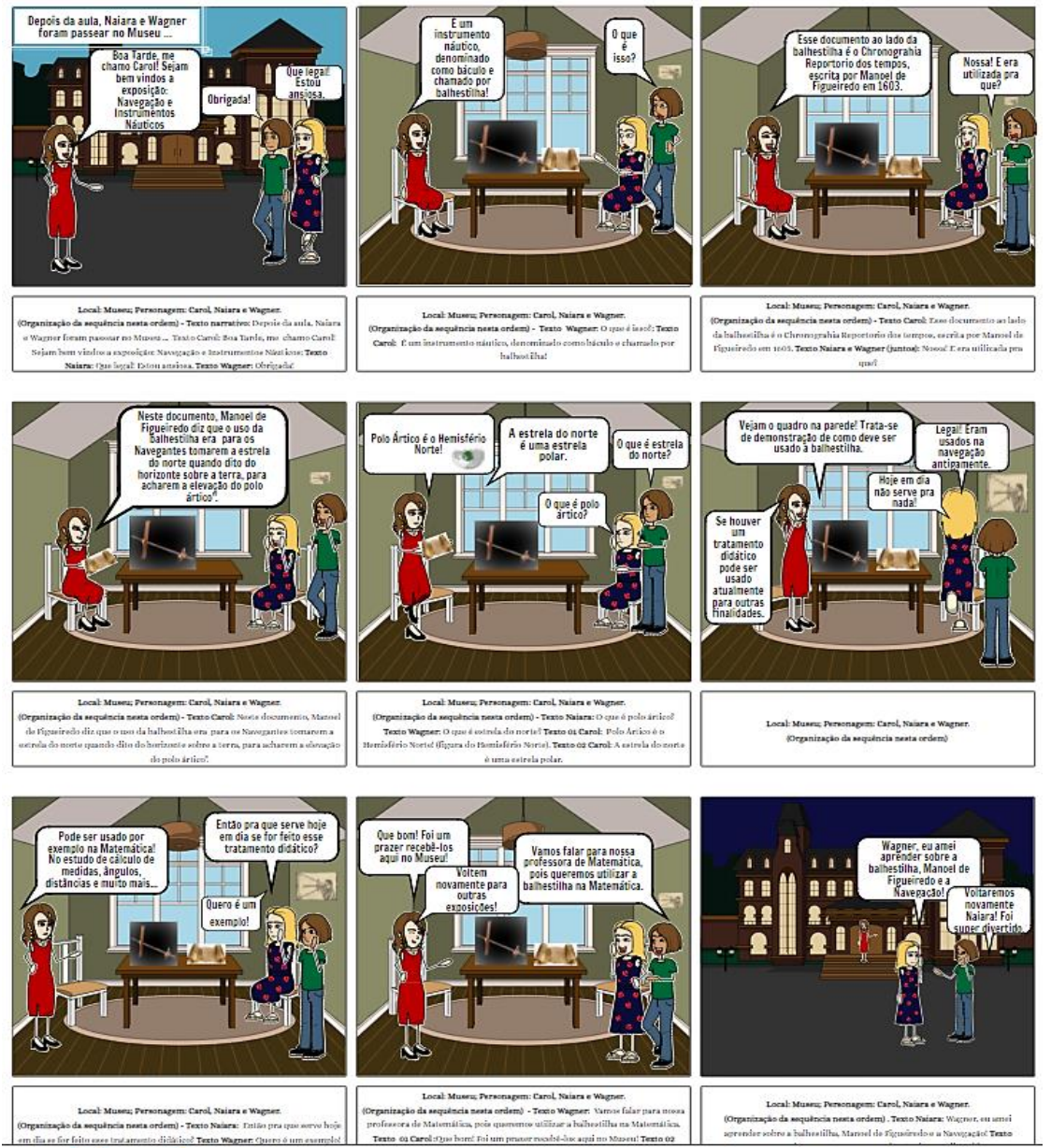

Fonte: Produzido pelas autoras na plataforma Storyboard That

Posteriormente, ao ser realizado a experimentação, temos a fase de Análise a posteriori e Validação, que conforme engenharia didática de segunda geração, obedece ao momento de avaliação dos dados coletados, em que confronta-se essa com a concepção e análise a priori das situações didáticas, para verificar se levantamos respostas para a pergunta diretriz, se alcançamos o objetivo geral e se validamos a experiência.

Diante disso, vale apontar que esse estudo validou-se, visualizando que as tecnologias do passado, no caso a balhestilha, submetida a uma transposição didática e as TDIC com o uso do Storyboard That para produção da História em quadrinhos "Navemática" comprovaram a aliança entre as tecnologias via investigação científica, em que, posteriormente será usado como roteiro para produção de um OA de história da Matemática a ser descrito em outras experiências, para uso futuro 
eISSN: $2526-9062$

em formação de professores de Matemática, que são importantes sujeitos no processo de ensino e aprendizagem dos alunos.

\section{Discussão de dados}

Entre as fases realizadas durante a pesquisa, destaca-se ainda nos momentos iniciais, na Análise Preliminar e Concepção e análise a priori das situações didáticas, o momento em que identificamos recursos que nos subsidiaram nesse processo, tendo o reconhecimento do instrumento a ser observado como tecnologia do passado, a TDIC como tecnologias do século XXI e a investigação cientifica de todos esses elementos que propuseram a aliança entre estes.

A comprovação mediante aos estudos na plataforma $J S T O R$, com a visualização de imagens antigas, que demonstram o uso e produção de instrumentos matemáticos, que eram um dos ofícios do passado, dos praticantes das matemáticas, consoante a Saito (2015) e que através destes a sociedade tinha mais facilidade nas ações do cotidiano, transformando assim, o meio e o homem de cada período histórico.

E, assim, entre os achados que validavam e demonstravam a importância dos instrumentos matemáticos como tecnologia dos passado, temos a (Figura 02), levantada no Science Museum Group, através da plataforma JSTOR, em que mostrava em uma loja na Barlett Street, Bath uma imagem de propaganda, com a seguinte descrição, J. Abraham, oftalmologista e fabricante de instrumentos matemáticos, sendo visualizado alguns destes, como sextante, telescópio, microscópio de suporte, bússola, globo, logotipos e brasão de armas.

Figura 02 - J. Abraham, oftalmologista e fabricante de instrumentos matemáticos

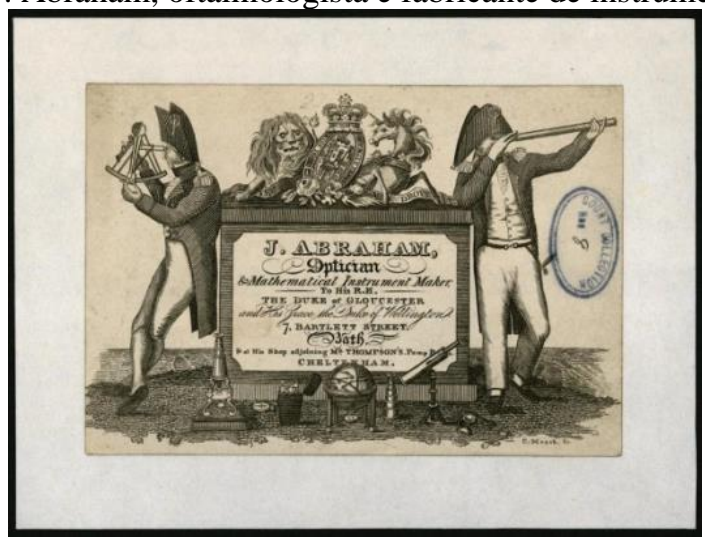

Fonte: < https://www.jstor.org/stable/10.2307/community.26288024>. 
eISSN: $2526-9062$

E, além disso, vale destacar que entre as ferramentas selecionadas para a transposição didática no ambiente digital, tivemos a Pixton e Storyboard That, no entanto, optamos pelo Storyboard That, que nos forneceu mais recursos gratuitamente, como cenas, personagens e demais funcionalidades.

E, ademais, observou-se a validação dessa pesquisa, pois ao confrontarmos as fases Concepções e análise a priori das situações didáticas com a Análise a posteriori, consegue-se verificar que a investigação científica de recursos, literaturas, plataformas de uso e produção de OAs, oportunizaram identificar e compreender a aliança entre as tecnologias do passado, instrumentos matemáticos, e, as tecnologias digitais, nesta ocasião, os OAs, a História em quadrinhos, como ferramenta facilitadora e promotora de ações significativas no ensino e aprendizagem de Matemática.

\section{Considerações Finais}

Concluímos que a necessidade humana por utilizar recursos tecnológicos já datam de séculos passados, que conforme a sociedade, costumes, episteme do período e práticas cotidianas, foram desenvolvendo, o que Veraszto et al. (2009) destacou por a razão do saber fazer, as atividades mediante aos utilitários tecnológicos existentes, podendo estes serem reconhecidos em distintas nomenclaturas de seus contextos, como utensílios, ferramentas, instrumentos, TIC ou ainda TDIC.

Nesse intuito, Souza (2020) nos conduz a perceber que a aliança entre tecnologias do passado e TDIC via investigação científica é significativa para o ensino de Matemática de variados assuntos ditos por "difíceis", mas que podem ser facilitados por essa articulação. Tendo ainda, a percepção de que através de uma formação potente de professores de Matemática, em caráter inicial ou continuado, sobre tal abordagem, podemos potencializar a aprendizagem de saberes matemáticos, contribuindo também, para a realização de uma práxis transformadora.

E, consoante a Wiley (2000) e Castro Filho et al. (2008), percebemos que a História em quadrinhos, produzida para essa experiência, além de permitir a aliança, através do tratamento didático de tecnologias do passado com as tecnologias digitais, nos inquietou a buscar em futuras experiências, usar esse recurso como um roteiro de produção para a construção de OA de história da Matemática.

Por fim, visualizou-se que o uso do instrumento do século XVII, balhestilha, transposto didaticamente para a interface de TDIC, pode ser ferramentas relevantes no ensino de conceitos matemáticos. E, diante do tratamento didático atribuído a este, observou-se, por exemplo, potencial 
eISSN: $2526-9062$

para o ensino de trigonometria, como recurso introdutório, através da utilização das Histórias em quadrinhos, que são OAs facilitadores, ou ainda, na inserção do instrumento, em outra tipologia de TDIC, sendo usado em séries finais do ensino fundamental ou no ensino médio.

\section{Referências}

ALMOULOUD, S. A; SILVA, M. J. F. Engenharia Didática: evolução e diversidade. Revemat: R. Eletr. de Edu. Matem. Florianopólis, v. 7, n.2, p. 26 - 27, 2012.

ALVES, V. B. Um estudo sobre os conhecimentos matemáticos mobilizados no manuseio do instrumento círculos de proporção de William Oughtred. Dissertação de Mestrado do Programa de Pós-Graduação em Ensino de Ciências e Matemática. Fortaleza: Instituto Federal de Educação, Ciência e Tecnologia do Ceará. 2019.

ALBUQUERQUE, S. M. de. Um estudo sobre a articulação contida no Traité de Gerbert (1843) e o ensino na formação de professores de matemática. Dissertação de Mestrado do Programa de Pós-Graduação em Ensino de Ciências e Matemática. Fortaleza: Instituto Federação de Educação, Ciência e Tecnologia do Ceará.

BATISTA, A. N. de S. Um estudo sobre os conhecimentos matemáticos incorporados e mobilizados na construção e no uso da balhestilha, inserida no documento Chronographia, Reportorio dos Tempos.... Dissertação de Mestrado do Programa de Pós-Graduação em Ensino de Ciências e Matemática. Fortaleza: Instituto Federação de Educação, Ciência e Tecnologia do Ceará. 2018.

BATISTA, A. N. de S., PEREIRA, A. C. C. A balhestilha (1603) como um instrumento matemático para o estudo de medidas na formação de professores de matemática. Acta Scientiarum.

Education, 43(1), e48188, 2020.

BRASIL. Ministério da Educação; Secretaria de Educação Básica. Base Nacional Comum Curricular. Brasília: MEC/ SEB, 2018.600 p.

CASTRO-FILHO, J.A; FREIRE, R. S; FERNANDES, A. C; LEITE; M.A. Quando objetos digitais são efetivamente para aprendizagem: o caso da matemática. In: XIX Simpósio Brasileiro de Informática na Educação - SBIE. Anais [...] Fortaleza, 2008.

FIORENTINI, D.; LORENZATO, S.. Investigação em educação matemática: percursos teóricos e metodológicos. São Paulo: Autores Associados, 2007.

MARTINS, E. B. Conhecimentos matemáticos mobilizados na manipulação das barras de calcular de John Napier descritas no tratado Rabdologiae de 1617. Dissertação de Mestrado do Programa de Pós-Graduação em Ensino de Ciências e Matemática. Fortaleza: Instituto Federação de Educação, Ciência e Tecnologia do Ceará. 2019. 
eISSN: $2526-9062$

OLIVEIRA, F. W. S. Sobre os conhecimentos geométricos incorporados na construção e no uso do instrumento jacente no plano de Pedro Nunes (1502-1578) na formação do professor de matemática. Dissertação de Mestrado do Programa de Pós-Graduação em Ensino de Ciências e Matemática. Fortaleza: Instituto Federação de Educação, Ciência e Tecnologia do Ceará. 2019.

OLIVEIRA, F. W. S.; PEREIRA, A. C. C. Indícios do Costume Relacionado a Divisão da Circunferência em Seus 360 Graus presente na Fabricação do Instrumento Jacente no Plano de Pedro Nunes. Revista Brasileira de História da Matemática, [S. 1.], v. 20, n. 39, p. 35-49, 2020.

PEREIRA, A. C. C.; SAITO, F. A reconstrução do Báculo de Petrus Ramus na interface entre história e ensino de matemática. Cocar, Belém, v. 13, n. 25, p.342-372, abr. 2019.

PEREIRA, A. C. C.; SAITO, F. Os conceitos de perpendicularidade e de paralelismo mobilizados em uma atividade com o uso do báculo (1636) de Petrus Ramus. Educação Matemática

Pesquisa: Revista do Programa de Estudos Pós-Graduados em Educação Matemática, [S.1.], v. 21, n. 1, abr. 2019.

PINHEIRO, A. C. M.; PEDROSA, V. N. M.; MENDONÇA, A. F. Uma proposta metodológica do uso do ambiente computacional como recurso didático para o ensino de conceitos matemáticos baseados na Sequência Fedathi. In: Encontro Nacional de Educação Matemática (ENEM). Anais [...] São Paulo, 2016.

RODRIGUES, A. M. M. Por uma filosofia da tecnologia. In: Grinspun, M.P.S.Z.(org.). Educação Tecnológica - Desafios e Pespectivas. São Paulo: Cortez, 2001: 75-129.

SAITO, F. As Matemáticas na Idade Média (Capítulo 4). In: História da Matemática e suas (re)construções contextuais. São Paulo: Ed. Livraria da Física/SBHmat, 2015, p. 159-193.

SANTOS, A. G. dos; OLIVEIRA, A. N. de; PEREIRA, A. C. C. As contribuições da régua de cálculo linear na construção dos saberes e das práticas docentes. Boletim Online de Educação Matemática, [S.L.], v. 8, n. 15, p. 17-36, 9 out. 2020.

SILVA, F. H. B. DA; PEREIRA, A. C. C. O báculo de Petrus Ramus e seu uso para medição de profundidade. Revista de Educação Matemática, v. 17, p. e020042, 2 set. 2020.

SOUZA, G. C. de. Aliança entre História da Matemática e Tecnologias via Investigação Matemática: reflexões e práticas. In: Souza, G.C. (Org.). Reflexões sobre aliança entre HM, TDIC e IM. São Paulo. Editora Livraria da Física, 2020.

VERASZTO, E. V.; SILVA, D. da.; MIRANDA, N. A.; SIMON, F. O. Tecnologia: Buscando uma definição para o conceito. Revista de Ciências e Tecnologias de Informação e Comunicação PRISMA.COM. 8, p. 19-46. 2009.

WILEY, D. A. Connecting learning objects to instructional design theory: a definition, a metaphor, and taxonomy. In: WILEY, D. A. (Ed.). The Instructional Use of Learning Objects. 2000. 